\title{
MRI and MR spectroscopy findings of the evolution of an intracranial germinoma: A case report
}

\author{
$\mathrm{JUN} \mathrm{LI}^{1}$, XUE-YAN ZHANG ${ }^{2}$, BIN WANG ${ }^{2}$ and $\mathrm{JUN}^{-Z U} \mathrm{GENG}^{1}$ \\ ${ }^{1}$ Department of Radiology, Yantai Affiliated Hospital of Binzhou Medical University, \\ Yantai, Shandong 264100; ${ }^{2}$ Nursing College, Binzhou Medical University, Yantai, \\ Shandong 264003, P.R. China
}

Received September 15, 2014; Accepted May 15, 2015

DOI: $10.3892 / \mathrm{ol} .2015 .3351$

\begin{abstract}
Intracranial germinomas (IG) are rare tumors that typically occur in the pineal gland and suprasellar region of the brain. IG can be successfully treated with radiation therapy and its recurrence is rare. The present study reports the case of a patient with IG involving the pineal and suprasellar regions. The patient was treated with radiotherapy, achieving significant remission. However, recurrence was discovered 10 months later. The patient was subsequently treated with chemotherapy and radiotherapy, resulting in gradual remission. The patient had recovered 2 months later and was in good health. The entire evolution process, including magnetic resonance (MR) imaging and MR spectroscopy findings, are described herein. The present case suggests that MR spectroscopy may be useful for monitoring the effects of treatment.
\end{abstract}

\section{Introduction}

Intracranial germinomas (IG) are rare tumors and account for $0.3-3.4 \%$ of all cases of intracranial cancer (1). Recurrence and dissemination is observed in $10-20 \%$ of cases (2). To date, $<100$ cases of IG with magnetic resonance (MR) spectroscopy findings have been documented. MR spectroscopy has gained importance in the evaluation of intracranial lesions and in the differential diagnosis of a number of tumor types, such as germinoma, teratoma, pineoblastoma and glioma (3). In IG, MR spectroscopy often reveals large lipid (Lip) peaks (3). IG can be successfully treated using radiation therapy and, thus, is the preferred treatment strategy for such patients.

Correspondence to: Mr. Jun Li, Department of Radiology, Yantai Affiliated Hospital of Binzhou Medical University, 717 Jinbu Street, Yantai, Shandong 264100, P.R. China

E-mail: bzmceducn@sina.com

Key words: evolution, intracranial germinoma, magnetic resonance imaging, magnetic resonance spectroscopy
Radiotherapy is associated with a good prognosis, with a 10 -year overall survival rate of $82.1 \%$ (4).

The present study reports a case of IG with recurrence 10 months after diagnosis. The patient underwent treatment with chemotherapy and radiotherapy. The entire evolution process, including MR imaging (MRI) and MR spectroscopy findings, are described herein. To the best of our knowledge, this is the first study to compare the metabolic changes of IG prior to and after treatment or recurrence.

\section{Case report}

In July 2013, a 25-year-old male patient presented to Yantai Affiliated Hospital of Binzhou Medical University (Yantai, China) with a 5-day history of headache and emesis. MRI revealed a large pineal mass measuring $2.2 \times 2.0 \times 2.4 \mathrm{~cm}$ (Fig. 1A), and proton MR spectroscopy of the lesion identified a relatively decreased $\mathrm{N}$-acetylaspartate (NAA) peak (12.0), an increased choline (Cho) peak (38.3) and a prominent Lip peak (11.3) at 1.3 ppm (Fig. 1B). The patient was diagnosed with a pineal germinoma based on MR and computed tomography findings, and received radiation therapy (33 Gy; 2 Gy/fraction, 5 fractions/week) almost immediately. After 20 days, the patient achieved significant remission, as observed by MRI (Fig. 1C). Furthermore, MR spectroscopy of the pineal lesion revealed that the NAA peak was increased (1.09) relative to the other peaks, with a decreased Cho peak (0.72) and no obvious Lip peak (0.12; Fig. 1D).

However, in May 2014, 10 months after the initial diagnosis, the patient was readmitted with the complaint of a headache. MRI revealed the presence of pineal and suprasellar masses (Fig. 1E). MR spectroscopy of the pineal mass identified changes similar to those observed in the initial examination (NAA, 13.0; Cho, 23.9, Lip, 12.1; Fig. 1F). Thus, a relapse of the germinoma was diagnosed. The patient received radiotherapy ( $40 \mathrm{~Gy} ; 2 \mathrm{~Gy} /$ fraction, 5 fractions/week) combined with two 3-week cycles of cisplatin $\left(60 \mathrm{mg} / \mathrm{m}^{2}\right.$, days $\left.3-4\right)$ and methotrexate $\left(300 \mathrm{mg} / \mathrm{m}^{2}\right.$, days $\left.1-2\right)$ chemotherapy, and gradually recovered; no lesions were visible 2 months later (Fig. 1G and H). The patient remained in good health at the most recent follow-up and all symptoms had resolved.

Written informed consent for the present study was obtained from the patient. 
A

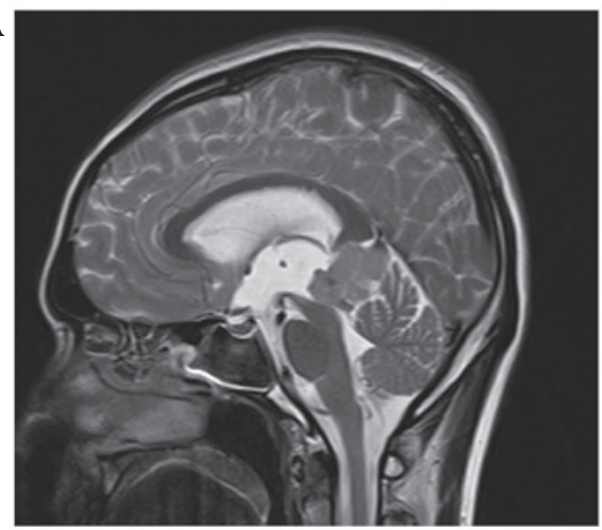

C

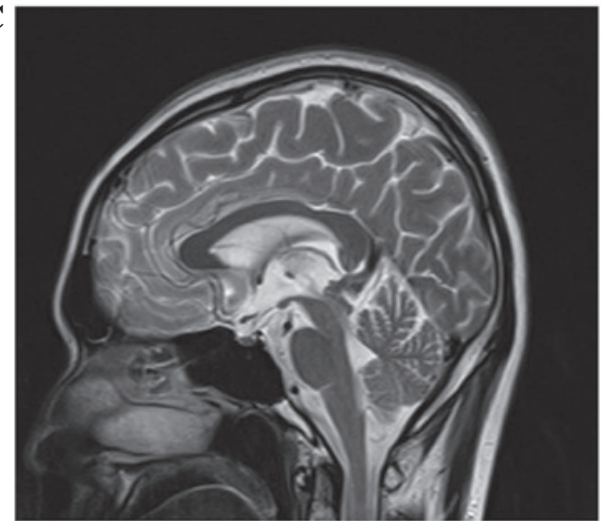

E

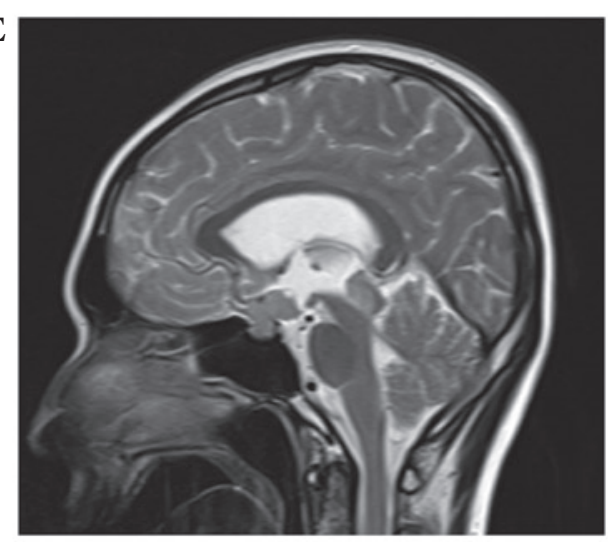

$\mathbf{G}$

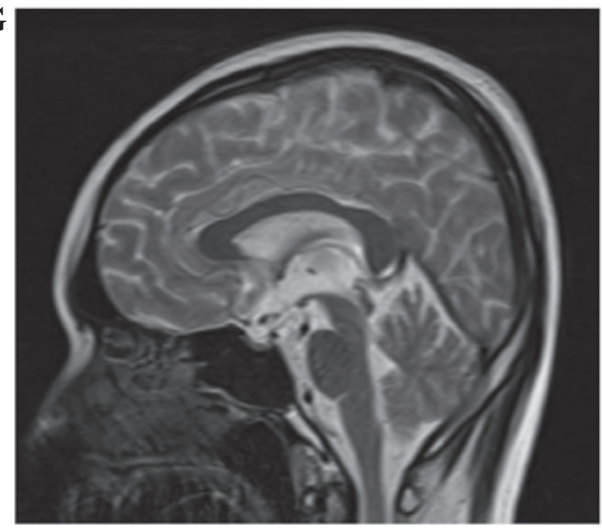

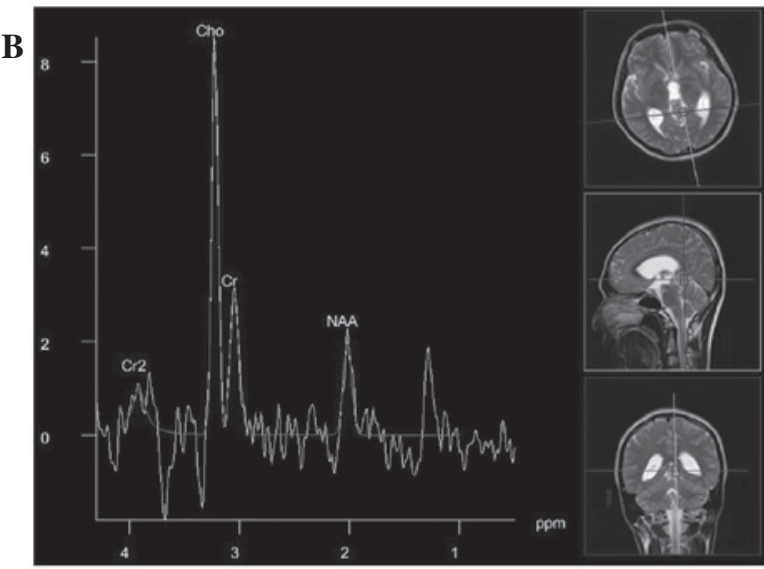

D

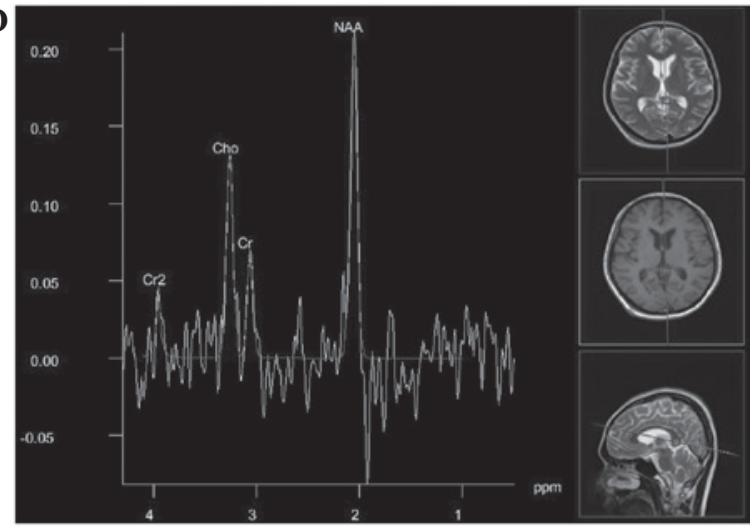

F
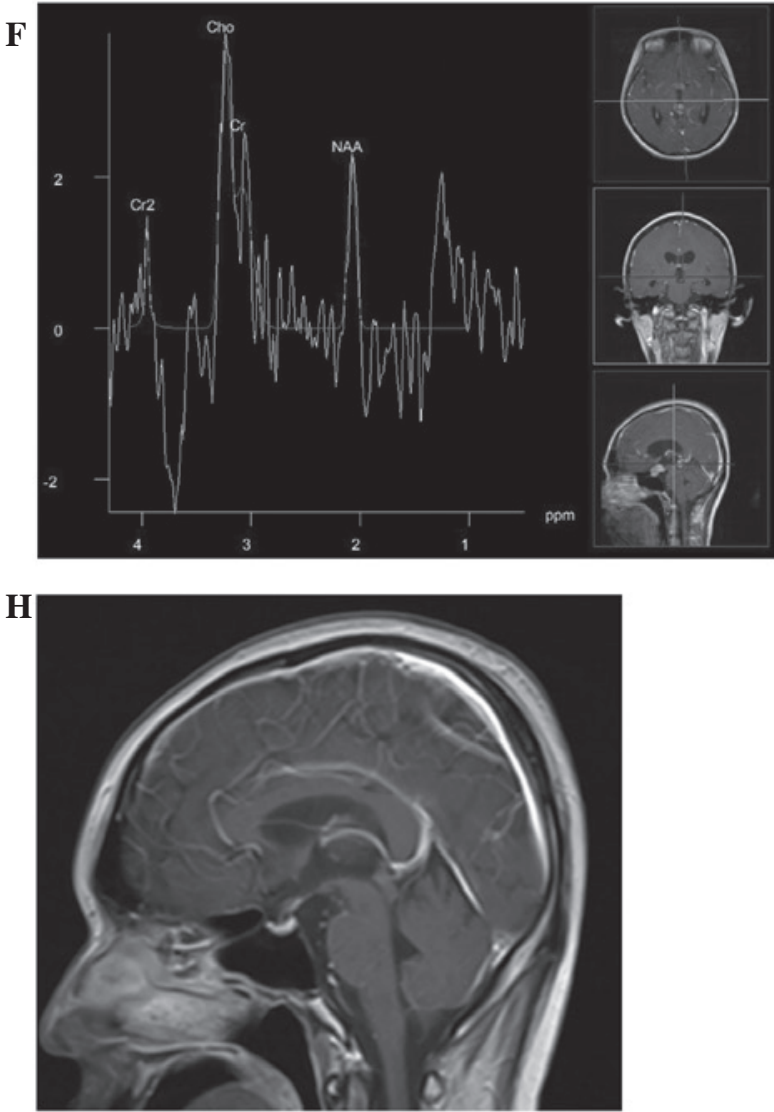

Figure 1.(A) Sagittal T2-weighted magnetic resonance imaging (MRI) revealing a large pineal gland mass.(B) MR spectroscopy demonstrating a low N-acetylaspartate (NAA) peak, a high choline (Cho) peak and an obvious lipid (Lip) peak. Following treatment with conventional radiotherapy, (C) sagittal T2-weighted MRI revealed a significantly reduced pineal mass, and (D) MR spectroscopy demonstrated an increased NAA peak, a decreased Cho peak and no obvious Lip peak. However, 10 months after the initial MRI examination, (E) sagittal T2-weighted MRI identified the presence of pineal gland and suprasellar masses. (F) Proton MR spectroscopy in the pineal mass revealed a low NAA peak, a high Cho peak and a prominent Lip peak. Following treatment with a combination of radiotherapy and chemotherapy, (G) sagittal T2-weighted MRI and (H) T1-weighted contrast-enhanced MRI indicated that all the lesions had disappeared. 


\section{Discussion}

Germinomas are tumors of germ cell origin that commonly occur intracranially in the pineal gland, posterior third ventricle and suprasellar regions (5). Suprasellar germinomas can be a metastatic deposit from a pineal region tumor or may primarily arise from the suprasellar cistern region. Synchronous lesions in the pineal gland and suprasellar region have been reported in $16-21 \%$ of cases (6). The treatment protocols vary; however, they are primarily radiation-based due to the acute radiosensitivity of this tumor type, with radiation therapy able to successfully treat $90 \%$ of patients with pure IGs (7). Recurrence of IG is observed in $10-20 \%$ of cases (2). In the case presented in the current study, it is proposed that the relapse of IG may be associated with an inadequate initial radiotherapy dose, as these tumors generally do not recur following adequate initial treatment $(8,9)$.

In recent years, MR spectroscopy has gained importance in the evaluation of intracranial lesions. The NAA peak is accepted as a neuronal marker. In normal conditions, the NAA peak is the highest, followed by the Cho peak (3). The NAA peak is commonly decreased in intracranial tumors. The Cho peak reflects the turnover of the cellular membrane, thus, an increased Cho peak indicates abnormal cell proliferation. This peak is predominantly increased in brain tumors (3). Lip peaks, which resonate at 0.9-1.3 ppm, are known to increase in highly malignant tumors, lymphocytes and tuberculosis (10). MR spectroscopy can help to diagnose IG through the appearance of the Lip peak. In the present study, MR spectroscopy revealed a decreased NAA peak, an increased Cho peak and a prominent Lip peak in IG. Following radiotherapy, the initial abnormalities detected on MR spectroscopy were found to have been reversed by the treatment; these findings were in agreement with the findings of previous studies. Thus, the ability of MR spectroscopy to sensitively detect metabolic abnormalities in germinoma may be useful for monitoring the effects of treatment from a biochemical perspective. In addition, MRI plays an important role in the diagnosis of IG. Through MRI and MR spectroscopy, the location, number, size and metabolic abnormalities of the tumor can be displayed.

In conclusion, MR spectroscopy is a useful non-invasive technique that provides important information regarding patients with IG and allows the outcomes of treatment strategies to be evaluated effectively.

\section{References}

1. Cuccia V and Galarza M. Pure pineal germinomas: Analysis of gender incidence. Acta Neurochir (Wien) 148: 865-871, 2006.

2. Janjetovic S, Bokemeyer C, Fiedler W, et al: Late recurrence of a pineal germinoma 14 years after radiation and chemotherapy: A case report and review of the literature. Onkologie 36: 371-373, 2013.

3. Tong T, Zhenwei Y and Xiaoyuan F. MRI and 1H-MRS on diagnosis of pineal region tumors. Clin Imaging 36: 702-709, 2012

4. Martens T, Rotermund R, Zu Eulenburg C, Westphal M and Flitsch J: Long-term follow-up and quality of life in patients with intracranial germinoma. Neurosurg Rev 37: 445-450, discussion 451, 2014.

5. Alapetite C, Brisse H, Patte C, et al: Pattern of relapse and outcome of non-metastatic germinoma patients treated with chemotherapy and limited field radiation: The SFOP experience. Neuro Oncol 12: 1318-1325, 2010.

6. Liang L, Korogi Y, Sugahara T, et al: MRI of intracranial germ-cell tumours. Neuroradiology 44: 382-388, 2002.

7. Calaminus G, Bamberg M, Baranzelli MC, et al: Intracranial germ cell tumors: A comprehensive update of the European data. Neuropediatrics 25: 26-32, 1994.

8. Wenger M, Lövblad KO, Markwalder R and Taub E: Late recurrence of pineal germinoma. Surg Neurol 57: 34-39, discussion 39-40, 2002.

9. Saito R, Kumabe T, Kanamori M, et al: Early response to chemotherapy as an indicator for the management of germinoma-like tumors of the pineal and/or suprasellar regions. J Clin Neurosci 21: 124-130, 2014.

10. Kaminogo M, Ishimaru H, Morikawa M, Suzuki Y and Shibata S: Proton MR spectroscopy and diffusion-weighted MR imaging for the diagnosis of intracranial tuberculomas. Report of two cases. Neurol Res 24: 537-543, 2002. 